\title{
Traumatic Brain Injury and Intracranial Hemorrhage Induced Cerebral Vasospasm and Delayed Cerebral Ischemia in a Patient with a Fall - Significance of the High-Cost Tertiary Investigations in Medico-Legal Practice; a Case Report
}

\author{
Raveendran $\mathrm{S}^{1 *}$, Vidanapathirana $\mathrm{M}^{2}$ \\ ${ }^{1}$ Office of the Judicial Medical Officer, Colombo South Teaching Hospital, ${ }^{2}$ Department of Forensic Medicine, \\ Faculty of Medical Sciences, University of Sri Jayewardenepura, Sri Lanka
}

\begin{abstract}
Delayed morbidity and mortality following traumatic and spontaneous intracranial haemorrhages (ICH) are common in both neurosurgical units and autopsy rooms. However, the causes of the delayed morbidity and mortality are complications of prolonged bed care such as aspiration or hypostatic pneumonia, urinary tract infection, dehydration, electrolyte imbalance, malnutrition and sepsis following bed sores. It can also be caused during the conservative management up to 14-21 days by delayed vasospasm and cerebral ischemia. A 67-year-old man with a history of hypertension was admitted to an ETU following a fall backwards. He lost his consciousness immediately after the fall for less than five minutes with retrograde amnesia, right sided paralysis and vomiting. On admission, blood pressure was $200 / 100 \mathrm{mmHg}$ and random blood sugar was $117 \mathrm{mg} / \mathrm{dl}$. Initial CT-brain revealed bilateral frontal lobe contusions with subarachnoid haemorrhages. In the second CT, an isolated left sided ICH that was not continuous with the brain surface was detected and the right sided hemiplegia was improved following evacuation. However, after 48 hours, he developed left sided hemiplegia. Repeat CT-brain, CT-angiogram and DSA-scan revealed no new hemorrhages, infarction, necrosis etc. There were no external injuries on the body. However, the MRI brain, showed a localized oedema of right supra-callosal area. Contre-coup contusions identified by CT-scan were compatible with the given history of fall backwards. Isolated left ICH identified in the second CT was compatible with a spontaneous ICH resulting right sided hemiplegia and could have precipitated the fall backwards. This ICH may be due to rupture of vessels due to long standing hypertension. The delayed left sided hemiplegia could have been due to the oedema of right supra-callosal area probably initiated by delayed cerebral ischemia fallowing vasospasm. This case also highlights the importance of radiological investigations in clinical forensic medicine for reconstruction of the events.
\end{abstract}

Keywords: ICH, traumatic, CT-Scan, fall, blood

Received: 02 Nov 2019, Revised version accepted: 20 Dec 2019, Published: 31 Dec 2019. *Corresponding author: Raveendran S, \Email: sathasivamraveendran10@gmail.com, iD https://orcid.org/0000-0002-2313-6491

Cite this article as: Raveendran S, Vidanapathirana M. Traumatic Brain Injury and Intracranial Hemorrhage Induced Cerebral Vasospasm and Delayed Cerebral Ischemia in a Patient with a fall - Significance of the High-Cost Tertiary Investigations in Medico-Legal Practice; A Case Report. Medico-Legal Journal of Sri Lanka, 2019;7(2):60-64

DOI: http://doi.org/10.4038/mljsl.v7i2.7401

Copyright: @ 2019 with the Medico-legal Journal of Sri Lanka.

This is an open-access article distributed under the terms of the Creative Commons Attribution 4.0 International License, which permits unrestricted use, distribution and reproduction in any medium provided the original author and source are credited.

\section{Introduction}

Sudden and delayed death and morbidity due to traumatic head injury and spontaneous intracranial haemorrhages are common in day to day practice in neurosurgical unit and autopsy rooms. ${ }^{[1]}$ Traumatic head injury includes subdural haemorrhage, extradural haemorrhage, subarachnoid haemorrhage, intracerebral haemorrhage, diffuse axonal injury, and contusions and lacerations of brain. ${ }^{[2]}$ Natural causes of intracranial haemorrhages are hypertension, infections, bleeding disorders (haemophilia, thrombocytopenia, DIC), ruptured aneurism, arterio venous malformations, congenital anomalies of blood vessels (cavernous malformation), Vascular tumors (angioma), intra cranial primary and secondary tumors (malignant) of the brain and drugs (thrombolytic agents, enoxaparin and warfarin and cocaine). ${ }^{[3]}$ Traumatic causes of intracranial haemorrhages should be differentiated from the natural causes in forensic practice. ${ }^{[1]}$ Sometimes, it is a difficult task in clinical 
cases when both conditions co -exist. It is extremely difficult when lack of external injuries and the unavailability of the tertiary investigation facilities.

\section{Case report}

A 67-year-old man was admitted to the ETU department of peripheral hospital with sudden fall on backwards while he was standing from the bed after sleeping. History revealed that he had immediate loss of consciousness for less than five minutes, retrograde amnesia of the event and severe vomiting. He had past history and family history of diabetes, hypertension and ischemic heart disease. He also had taken Aspirin $75 \mathrm{mg}$ as a routine. He was transferred to a tertiary care hospital for further management due to the deterioration in the GCS scores.

BHT revealed that on admission, his GCS was 14 , blood pressure was $200 / 100 \mathrm{mmHg}$, random blood sugar was $117 \mathrm{mg} / \mathrm{dl}$ and ECG was normal. Neurological examination was unremarkable on admission except confused verbal response. Initial Non-contrast CT-brain (Figure 01) revealed that small bilateral frontal lobe contusions and thin subarachnoid haemorrhage but no cerebral oedema. His GCS level was reduced to 11-12 and developed right hemiplegia with aphasia at eight hours after the admission. Repeat CT-scan (Figure 02) revealed an isolated left intracerebral haemorrhage (ICH) near the supracallosal region.

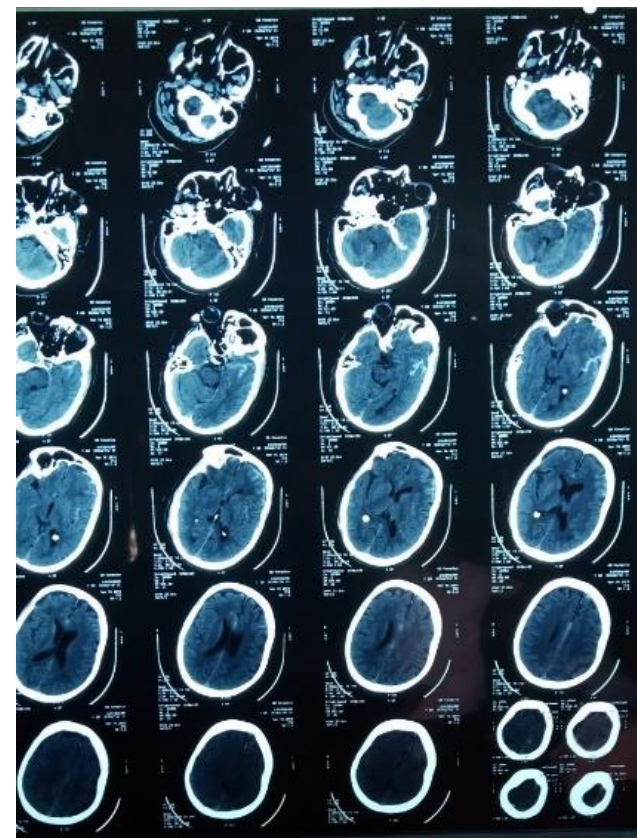

Figure 01: Initial Non-contrast CT brain: Small bilateral frontal lobe contusions and overlying thin subarachnoid haemorrhage

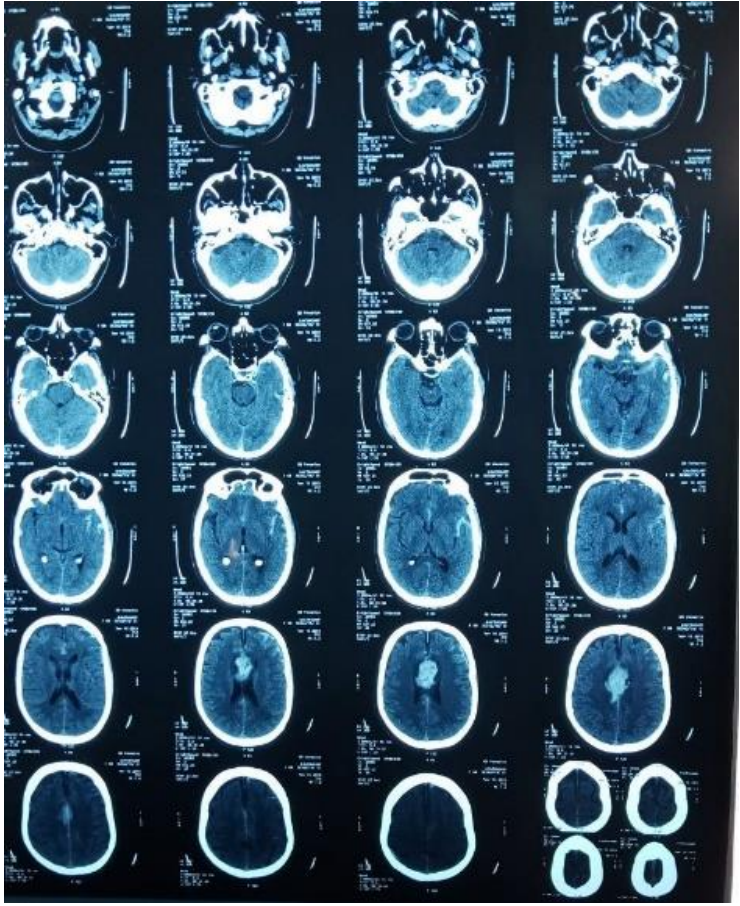

Figure 02: Repeat CT-scan: An isolated left intracerebral haemorrhage (ICH) near the supracallosal region

Patient underwent CT-angiogram (Figure 03) and digital subtraction angiography (DSA) of the brain (Figure 04) that revealed no aneurisms, rupture of main blood vessels or vascular malformations. He was medically managed and he was undergone evacuation of hematoma by craniotomy. Recovery was uneventful. Right side hemiplegia was improved.

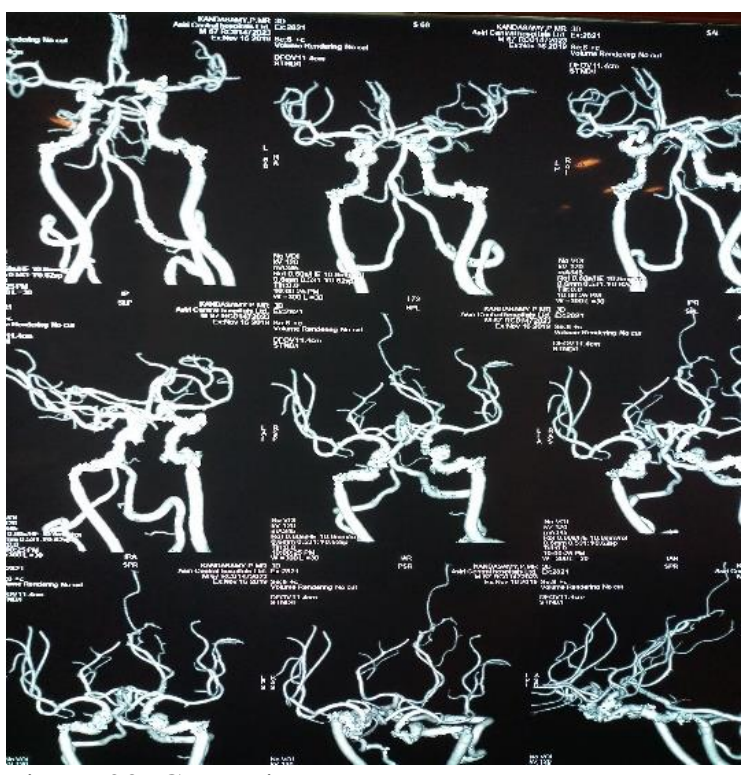

Figure 03: CT-angiogram 


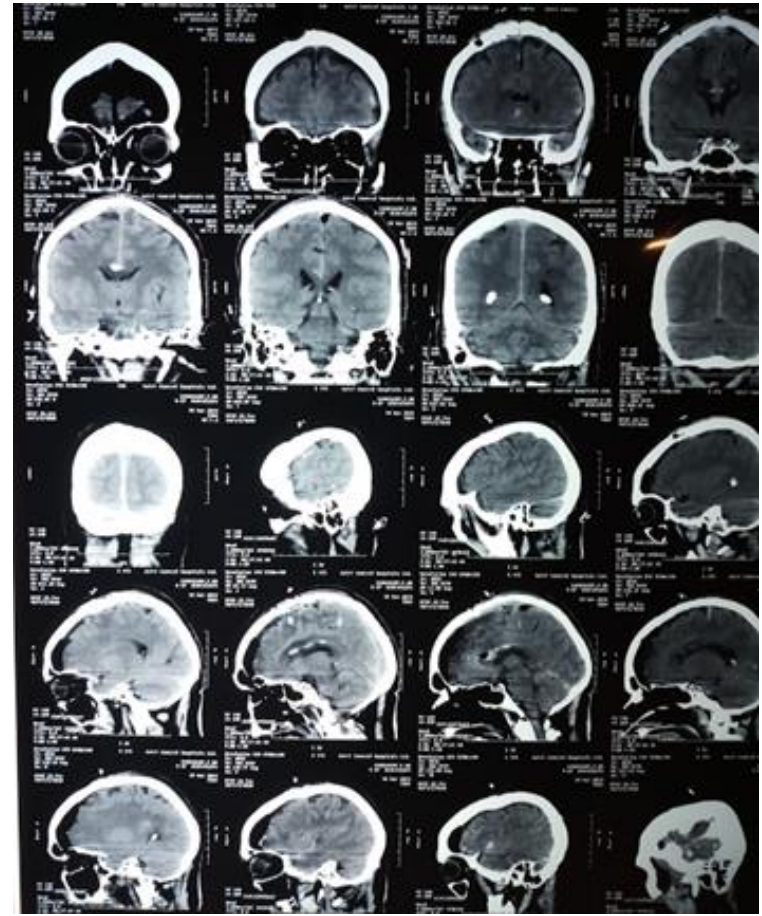

Figure 04: Digital subtraction angiography [DSA] of the brain.

Figure 03 (CT-angiogram) and Figure 04 (Digital subtraction angiography [DSA] of the brain) revealed absence of aneurisms, rupture of main blood vessels or vascular malformations.

After 48 hours he developed paralysis of left upper limb and lower limb. Repeat Non-contrast CT-brain, CT-angiogram (Figure 05) and DSA-scan (06) revealed no new hemorrhages, infarctions or necrosis. But MRI brain revealed oedema of the brain near to callosal region.

Figure 05 (Repeat CT-angiogram) and Figure 06 (Repeat DSA-scan) revealed no new hemorrhages, infarctions or necrosis.

Medico- legal examination revealed no external injuries on the body. Original clothing was not available for the examination. He was managed in ICU with prolonged bedridden care, insulin, a calcium channel blocker nimodipine, GTN infusion, noradrenaline and intravenous antibiotics. Aspirin and the antiplatelet medication clopidogrel were omitted. His blood pressure maintained at $190 / 110 \mathrm{mmHg}$ in the ICU to maintain the perfusion pressure. His aphasia and left sided weakness was slightly improved on $12^{\text {th }}$ day of admission.

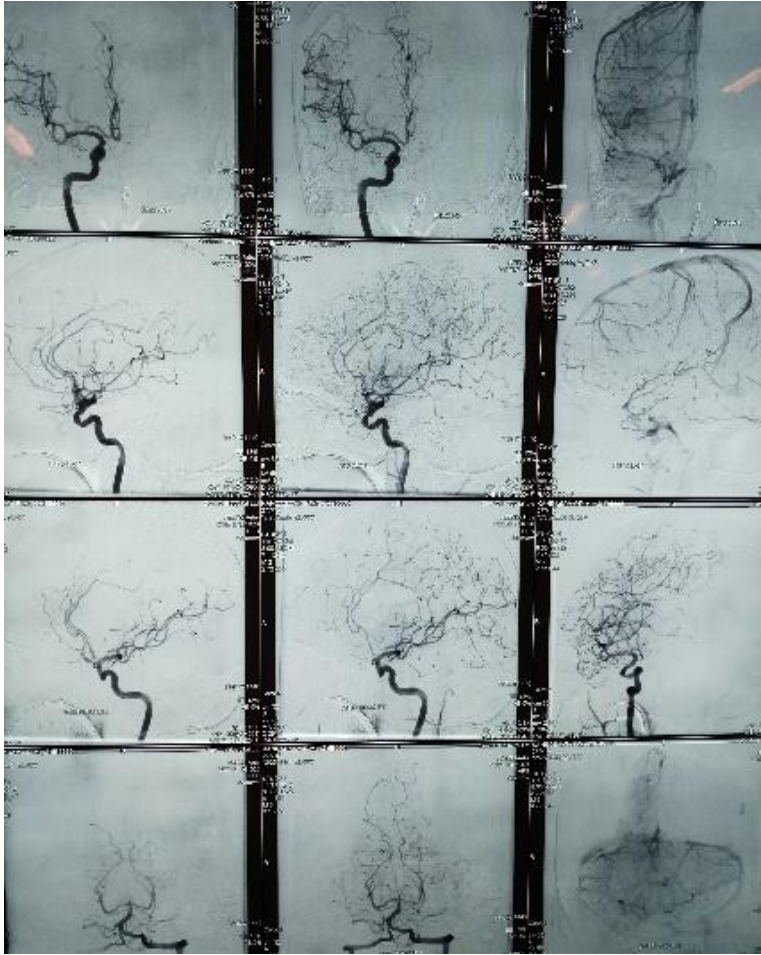

Figure 05: Repeat CT-angiogram

Medico- legal examination form was issued by the police according to the request made by ward doctors, because of the suspicious circumstances due to missing of the bystander during the ward stay.

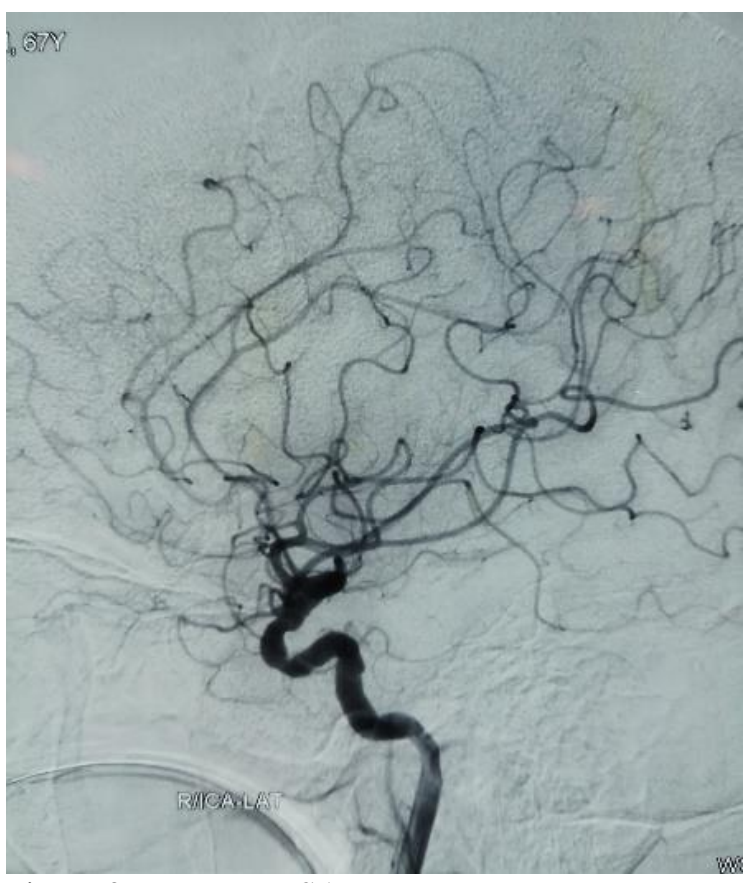

Figure 06: Repeat DSA-scan. 


\section{Discussion}

He did not have external injuries on the body. Only had bilateral small frontal contusion, subarachnoid haemorrhage and isolated intracerebral hemorrhage. Traumatic origin of intra cranial injuries should be differentiated from not traumatic injuries for medicolegal purpose. Even he had no external injuries to compare, the CT evidence of bilateral frontal lobe contusions (contra-coup injuries) and subarachnoid haemorrhages were compatible given history of fall backwards. ${ }^{[4]}$ This is compatible with earlier studies that one can develop head injury by intentional and unintentional fall through his own height. ${ }^{[4]}$

There were no external injuries. It may be due to interference by cloths, scalp hair and /or force generated was not enough to cause tissue damage or skull fracture.

Even he had a no aneurism, ruptured blood vessels or vascular malformations on CT angiography and DSA scan, ICH detected on the second CT was isolated in location and it was not continuous with surface indicates that it is unlikely to be originated from the trauma. ${ }^{[1]}$ It may be due rupture of weaken small vessels related to long standing hypertension which is very high on admission. ${ }^{[5]}$ It may be aggravated by blood thinning agents. ${ }^{[3]}$

His right side weakness was recovered after evacuation of ICH by craniotomy, but he developed left side hemiplegia after 48 hours while he was in the ICU. Repeated radiological images excluded atherosclerotic / thrombotic occlusion of blood vessels ${ }^{[6]}$, new haemorrhages, repeated bleeding, brain infarction/ necrosis, venous sinus thrombosis, tumor and infection of brain and meninges ${ }^{[7]}$ except oedema on right cerebrum in localized area near to supra callosal area. According to the neurosurgeon, the left side weakness may be due to delayed cerebral ischemia fallowing vasospasm. ${ }^{[8,10]}$ Other possible explanation was oedema near to corpus callosum compressing the fibers in the white matter.

Theoretically ICH can cause vascular spasm within 714 days, but can extended up to twenty-one (21) days. ${ }^{[9]}$ It is common in younger persons with ICH than older. ${ }^{[9,10]}$ If vascular spasm involves that artery supplying to vital Centre of the brain can cause sudden morbidity or death at any point even though amount of ICH is not fatal. It can be demonstrated in clinical cases but almost impossible in autopsy cases like cardiac Reynaud phenomenon. ${ }^{[5]}$

This case revealed traumatic frontal lobe contusion and SAH and non-traumatic ICH. Commonly SAH at the base of the brain is causing the vascular spasm. But delayed spasm can occur commonly with SAH at any places. ${ }^{[10]}$ It is less likely with intra cerebral hemorrhage. ${ }^{[10]}$ If vascular spasm caused by spontaneous ICH, criminal liability may be less when traumatic and non-traumatic ICH co-exist and it may be significant when considering the insurance and compensation. In this case it is difficult to find the cause of vascular spasm whether it was caused by nontraumatic ICH or Traumatic ICH. It may be very significant in the case of non-accidental falls especially in assaults.

This patient had traumatic ICH without the evidence of external injuries on body, scalp and skull bones. This is compatible with earlier studies that one can develop head injury without any external evidence of fall. ${ }^{[4]}$

Interpretation of the findings is very important to reconstruct the events medico legally. It will aid the legal system to implement the justice in significant cases when traumatic and non-traumatic ICH co-exist. (a) Whether traumatic ICH was preceded the spontaneous ICH or vice versa? (b) This patient developed left sided hemiplegia after the craniotomy at 48 hours of admission. Whether it was caused by traumatic ICH or spontaneous ICH?

Even spontaneous ICH was visible on second non contrast CT which was taken after 10 hours of first CT. Spontaneous ICH might developed just before the fall which was not visible on first CT scan and it may be the reason for the fall. With time haemorrhage may be expanded with the additional contribution of Aspirin and it was visible on the second CT scan.

He already had ischemic heart disease and diabetes mellitus which were under control with medication. His random blood sugar was $117 \mathrm{mg} / \mathrm{dl}$ and ECG was normal on admission. These findings rule out the hypoglycemia and cardiac causes as a cause for the sudden fall most probably.

The possibility of stress induced hypertensive ICH fallowing fall cannot be ruled out completely, but it is a remote possibility.

He may have developed left sided hemiplegia after 48 hours most probably due to delayed cerebral ischemia fallowing subarachnoid haemorrhage ${ }^{[9,10]}$ But it might be due to fallowing intracerebral haemorrhage and /or surgical/radiological intervention. ${ }^{[10]}$ Vasospasm was managed with nimodipine and nor-adrenaline ${ }^{[11]}$ and also by maintaining the blood pressure at higher level to sustain the perfusion pressure. His aphasia and left 
sided weakness was improved on $12^{\text {th }}$ day which may support the theory of delayed cerebral ischemia fallowing subarachnoid haemorrhage. This finding explain the delayed mortality and morbidity in a patient with traumatic and non-traumatic intracranial haemorrhages.

Most of the findings were detected and events are reconstructed medico-legally with the help of tertiary investigations such as CT scan -non contrast brain, angiography of brain, DSA scan and MRI brain. It highlights that importance of radiological investigations in Forensic practice in addition to the clinical management.

Analysis of the intra cranial hemorrhages with radiological investigations and medico legal examination confirmed that given history of nonintentional fall by close eye witness and excluded the foul play.

\section{Conclusions}

Contre-coup contusions identified by CT-scan were compatible with the given history of fall backwards. Isolated left sided ICH identified in the second CTscan was compatible with a spontaneous ICH resulting right sided hemiplegia and could have precipitated the fall backwards. This ICH may be due to rupture of vessels due to long standing hypertension. The delayed left sided hemiplegia could have been due to the oedema of right supra-callosal area probably initiated by delayed cerebral ischemia fallowing vasospasm. This case highlights the importance of radiological investigation in clinical forensic medicine for reconstruction of the events and also confirms the previous studies of delayed cerebral ischemia following traumatic brain injury and intracranial hemorrhage.

\section{Disclosure statement}

Conflicts of interests: The authors declare that they have no conflicts of interests.

Funding: None

\section{References}

1. Spitz WU. Trauma of the nervous system. In: Spitz and Fisher's Medico legal investigation of death.4th ed. USA: Charles C Thomas Publishers Ltd; 2005. pp. 994-1077.

2. DiMaio VJ, DiMaio D. Trauma to the skull and brain; craniocerebral injuries, Forensic Pathology, 2nd ed. New York: CRC press; 2001. pp. 101-41

3. Fewel ME, Thompson BG, Hoff JT: Spontaneous Intracerebral Hemorrhage: a review. Neurosurg Focus 15: 2003.

4. Saukko P, Knight B. Head and spinal injuries. In: Knight's Forensic Pathology.4th Ed. New York: CRC press; 2016. pp. 167-213

5. Richard NM. Blood vessels. In: Robbins and Cotran Pathologic basis of disease. 1st ed. India: Thomson Press ltd; 2015. pp. 483-22

6. Richard NM. Haemodynamic disorders, Thromboembolic disease, and shock. In: Robbins and Cotran Pathologic basis of disease. 1st ed. India: Thomson Press ltd; 2015. pp. 113-35

7. Frosch MP, Anthony DC, Girolami DU. The central nervous system. Blood vessels. In: Robbins and Cotran Pathologic basis of disease. 1st ed. India: Thomson Press ltd; 2015. pp. 1251318

8. Khatri, I. A., Verma, N., Alkawi, A., Janjua, N., \& Kirmani, J. F. Cerebral vasospasm in intracerebral hemorrhage-case report. Journal of vascular and interventional neurology. 2009;2(1):139-141.

9. Kochanowicz J, Lyson T, Lewszuk A, SytaKrzyzanowska .The incidence of vasospasm after intracerebral haemorrhage. Prog Health Sci. 2012;2(2):70-4.

10. Al-Mufti F, Amuluru K, Changa A, Lander M, Patel N, Wajswol E, Al-Marsoummi S, Alzubaidi B, Singh IP, Nuoman R, Gandhi .Traumatic brain injury and intracranial hemorrhage-induced cerebral vasospasm: a systematic review. Neurosurg Focus. 2017 Nov;43(5): E14. Doi: 10.3171/2017.8.FOCUS17431.

11. Wartenberg KE, Update on the Management of Subarachnoid Hemorrhage. Future Neurology. 2013;8(2):205-224. 